\title{
Fading Mitigation with Diversity Schemes in WCDMA: Technical Review
}

\author{
RajkumarGupta $^{1}$, Amit chaurasia $^{2}$, Ashwani yadav $^{3}$ Vaishali $^{4}$ \\ Assistant Professor, ASET, Amity University Rajasthan, Jaipur, India ${ }^{1,2,3,4}$
}

\begin{abstract}
Wide Code Division Multiple Access (WCDMA)a third generation wireless communication system that has high bandwidth and offers multimedia communication capabilities for global mobile communications but the performance of WCDMA is degraded with various factors such as interference, fading and scattering. Diversity is a technique which is used to mitigate the fading problem in wireless channel at a relatively low cost.This paper gives the review of different diversity techniques to mitigate the fading problem and for improving the overall performance of the communication system. In different diversity techniques, space diversity technique is an important technique and maximum ratio combining (MRC) is the most effective technique which is a part of space diversity technique.MRC produces an output signal to noise ratio (SNR) equal to the sum of the individual SNRs.
\end{abstract}

Keywords: WCDMA, Diversity Techniques, Selection Combining, Maximum Ratio Combining

\section{INTRODUCTION}

Wireless communication is the fastest growth period in the history of communication technologies. Advance Mobile Phone System (AMPS) was the first cellular system. It allocates bandwidth of $60 \mathrm{KHzto}$ each user. This allocation consisted of two $30 \mathrm{KHz}$ Channels: One is the uplink channel and another is downlink channel [1]. In the AMPS system spectrum is shared amongst users and this technology is called Frequency Division Multiple Access (FDMA) [2]. The first digital cellular phone was standard IS-54. It supported three users in each $30 \mathrm{KHz}$ of bandwidth. Which is used in AMPS system [3].

This improves the users three times per channel along with other advantages such as error-correction and security. This improvement in the number of supportable voice channels was due to speech coding, compression, digital modulation and time Division Multiple Access (TDMA). The multiple access schemes in GSM were TDMA structure that is implemented on multiple frequency subbands. Two frequency bands of $25 \mathrm{MHz}$ each at around $900 \mathrm{MHz}$ (GSM 900) had been allocated for the use in GSM: $890-915 \mathrm{MHz}$ for the uplink and $935-960 \mathrm{MHz}$ for the downlink. The GSM system in also operates in the $1800 \mathrm{MHz}(\mathrm{GSM}$ 1800) frequency band[4]. Each uplink and downlink GSM frequency band was divided into 124 pairs of carriers spaced by $200 \mathrm{kHz}$ and each carrier was divided into 8 time slots using TDMA[5][6]. In CDMA the narrowband message signal is multiplied with a large bandwidth signal that is called the spreading signal. In spread spectrum technique each users is multiply with pseudo-noise code sequence hence increase the bandwidth of users[7]. The pseudo-noise code sequence generates using shift register and repeat after known period. The CDMA system is two types: non-orthogonal and orthogonal. The TDMA, FDMA and orthogonal CDMA all have the same capacity in an additive noise channel[8]. In CDMA, each user uses one of the ten available orthogonal codes that can be transmitted all the time over all of the frequency.[9]. The increasing demand for internet and wireless services such as voice, video and data highlights the need of increase in the capacity of the system. The 3rd generation partnership project (3GPP) and 3rd generation partnership project two (3GPP2) had developed the wideband code-division multiple access (WCDMA) technologies and CDMA2000 respectively[10].

\section{WIDEBAND CDMA}

Wideband CDMA (WCDMA) is the second 3Gairinterfacestandard which is based on CDMA. It is an asynchronous scheme and it uses a $5 \mathrm{MHz}$ carrier frequency. The use of a wider carrier frequency is to provide high data rates. WCDMA is considered to be wideband technology which is based on the direct sequence spread spectrum transmission scheme. In direct sequence spread spectrum user information bits are spread over a wide bandwidth and user data multiplied with the quasi-random bits called chip. In order to support very high bit rates (up to $2 \mathrm{Mbps}$ ), the use of a variable spreading factor and multi code connection is required. In WCDMA the chip rate of 3.84 Mcps leads a carrier bandwidth of $5 \mathrm{MHz}$ [11].

\subsection{WCDMA KEY FEATURES}

The key features of the WCDMA are[12], [13]:

- It provides high service flexibility $\square \square$

- It supports the high data rate transmission: $2 \mathrm{mbps}$ for local area coverage and $384 \mathrm{kbps}$ for wide area coverage.

- Support of inter frequency hand over and hand over to other systems including hand over to GSM.

- Efficient packet access

- It supports both time division duplex (TDD and frequency division duplex (FDD)

\subsection{PROTOCOL ARCHITECTURE}

The design of modern wireless networks is based on a layered architecture and each layer is associated with specific functions and communicates with other layers through a particular interface. The advantage of this technique is that each layer can be redesigned without affecting the rest of the layers as long as it keeps the same 
interface[14]. Fig.1 shows the radio interface protocol

Model. The radio interface protocol architecture of WCDMA consists of the three layers [1].

- $\quad$ Radio physical layer (layer 1, L1)

- $\quad$ Radio data link layer (layer 2, L2)

- $\quad$ Radio network layer (layer 3, L3)

\subsection{W-CDMA RADIO PARAMETERS}

The main radio parameters that define in WCDMA radio interface are given in Table1 [1]

Fig.1 Radio Interface Protocol Model

Table 1 Radio parameter of WCDMA system[15]

\begin{tabular}{|c|c|c|}
\hline \multicolumn{2}{|c|}{ WCDMA } & PARAMETERS \\
\hline \multicolumn{2}{|c|}{ Channel bandwidth } & $5,10,20 \mathrm{MHz}$ \\
\hline \multicolumn{2}{|l|}{ Chip rate } & $3.84^{*}(1,2,4) \mathrm{Mcps}$ \\
\hline \multicolumn{2}{|c|}{ Multiple Access } & DS CDMA \\
\hline \multirow{2}{*}{ Frame length } & Variable-rate speech & 10 or $20 \mathrm{~ms}$ \\
\hline & Packet Data & $10-80 \mathrm{~ms}$ \\
\hline \multirow{5}{*}{ Modulation } & \multirow{3}{*}{ Spreading } & Balanced QPSK (downlink) \\
\hline & & QPSK (Uplink) \\
\hline & & Complex spreading circuit \\
\hline & \multirow{2}{*}{ Data } & QPSK (downlink) \\
\hline & & BPSK (Uplink) \\
\hline \multicolumn{2}{|c|}{ Coherent detection } & Pilot Symbol assisted \\
\hline \multicolumn{2}{|c|}{ Channel Coding } & Convolutional and turbo coding \\
\hline \multirow{2}{*}{\multicolumn{2}{|c|}{$\begin{array}{l}\text { Scrambling code } \\
\text { Interleaving }\end{array}$}} & $10 \mathrm{~ms}$ \\
\hline & & $10 / 20 / 40 / 80 \mathrm{~ms}$ \\
\hline \multicolumn{2}{|l|}{ Multirate } & Variable spreading and multicode \\
\hline \multicolumn{2}{|c|}{ Spreading factor } & $4-512$ \\
\hline \multirow{2}{*}{ Spreading } & Downlink & $\begin{array}{l}\text { Variable length orthogonal } \\
\text { sequences for channel separation. } \\
\text { Gold sequence } 2^{18} \text { for user and cell } \\
\text { separation }\end{array}$ \\
\hline & Uplink & $\begin{array}{l}\text { Variable length orthogonal } \\
\text { sequences for channel separation. } \\
\text { Gold sequence } 241 \text { for user } \\
\text { separation }\end{array}$ \\
\hline \multicolumn{2}{|c|}{ Inter base station timing } & Asynchronous \\
\hline
\end{tabular}

III. PROBLEMS ASSOCIATE WITH WCDMA

When a signal is transmitted from source to destination through the channel, there are some factors which degrade the performance of signal and these degradation factor are discuss here:

\subsection{PATH LOSS}

In a point-to-point wireless communication system, when a transmitter communicates with a receiver by sending an electromagnetic signal through a wireless medium, the strength of the signal attenuates as it traverses the medium and becomes weaker as the propagation distance increases. Path loss phenomenon is given by following equation[16].

$$
P_{r}=P_{0}\left(\frac{1}{4 \pi d / \lambda}\right)^{2}\left|1+a_{v} e^{j \Delta \Phi}\right|^{2}
$$

Where, $\mathrm{a}_{\mathrm{v}}=$ the reflection coefficient

$\Delta \Phi=$ the phase difference between a direct path and a reflected path

$\mathrm{P}_{\mathrm{o}}=$ the transmitted power

$\mathrm{d}=$ the distance

$\lambda=$ the wavelength

\subsection{NOISE}

It is an undesired signal in a communication system or unwanted disturbances superimposed on a useful signal which tends to obscure its information content.

\subsection{INTERFERENCE}

Interference is created due to another mobile in the same cell [17]. It is the major limiting factor in the performance of cellular system. It causes cross talk on voice channel. Interference is more in urban areas, due to the greater RF noise floor and the large number of base stations and mobiles. Co-channel interference and adjacent channel interference are the two types of cellular interference. Cochannel interference (intra-cell) is due to the signal from the other users in the home cell [18]. Co-channel interference is estimated by Carrier-to-interference ratio which is given below [16].

$$
\frac{C}{I}=\frac{1}{\sum_{K=1}^{K_{I}}\left(\frac{D_{K}}{R}\right)^{-\gamma}}=\frac{1}{\sum_{K=1}^{K_{I}}\left(q_{k}\right)^{-\gamma}}
$$

Where $q_{k}$ is the co channel interference reduction factor with $k$ th co channel interference cell

$q_{k}=\frac{D_{K}}{R}$

Where , $D=$ frequency reuse distance

$\mathrm{D}=(3 \mathrm{~K})^{1 / 2} \mathrm{R}$

$\mathrm{R}=$ Radius of cell

$\gamma=$ propagation path loss constant

\subsection{FADING}

In a typical wireless communication environment multiple propagation paths often exist from a transmitter to a receiver due to scattering by different objects. Signal copies following different paths can undergo different attenuation, distortions, delays and phase shifts. Constructive and destructive interference occurs and if destructive interference occurs, the signal power can be significantly diminished and the phenomenon is called fading [6].In wireless systems, fading may either be due to multipath propagation, referred to as multipath induced fading, or due to shadowing from obstacles affecting the wave propagation, sometimes referred to as shadow fading. In a wireless mobile system a signal can travel from transmitter to receiver through multiple reflective paths which is known as multipath propagation and is illustrated in Fig.2. To overcome the fading effect diversity technique is used[19]. Thus, the idea behind diversity is to send the same data over independent fading paths. These independent paths are combined in some way such that the effect of fading on the resultant signal is reduced.

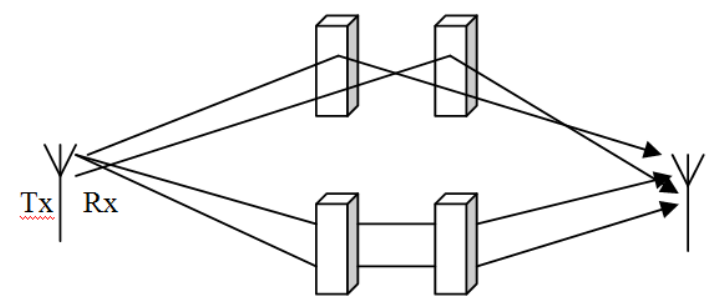

Fig. 2 Multipath Propagation 
IV. ERROR COMPENSATION TECHNIQUES

To remove the error in WCDMA can be used some error compensation techniques such as forward error correction, adaptive equalization, interference cancellation techniques and diversity techniques [20][21].

\section{DIVERSITY}

Diversity is the technique which is used to mitigate the fading problem in wireless communications systems due to multipath propagation. In the diversity if one signal path contains a deep fade at a particular point of time, in this condition there is a chance to get the strong signal in the another independent path.[22].

\subsection{SIMPLE DIVERSITY TECHNIQUES}

The different types of diversity techniques, used in wireless communication systems, are reviewed here:

\subsubsection{FREQUENCY DIVERSITY}

In frequency diversity the information signal is transmitted over multiple frequency slots and these frequency slots are separated by a bandwidth $\Delta f_{c}$ of the channel as shown in Fig.3 [23]. Spread spectrum modulation and orthogonal frequency division multiplexing (OFDM) are the frequency diversity techniques.

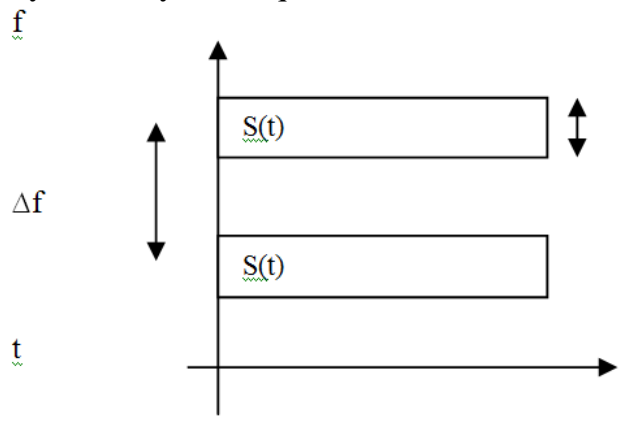

Fig.3Frequency diversity

When the coherence bandwidth of the channel is larger than the spreading bandwidth or equivalently, then spread spectrum techniques are useless. Xiaoya et.al [24] proposed the complex quadratic spreading sequences to achieve full frequency diversity order through maximum ratio combining (MRC) reception in the frequency domain. Li et.al [25] proposed a frequency diversity technique for blue tooth that used dual channel transmission to deal with both dynamic and static cochannel interferences.

\subsubsection{TIME DIVERSITY}

In time diversity the same information signal is transmitted in different time slots. The separation between the time slots has to be at least the coherence time $\Delta t_{c}$ as shown in Fig 4. The time interval depends on the fading rate and increases with the decrease in the rate of fading[6]. A coding structure known as interleaving is often used to realize time diversity where the receiver knows the code before any transmission takes place. Time interleaving, together with error correction coding can provide diversity improvement[26].

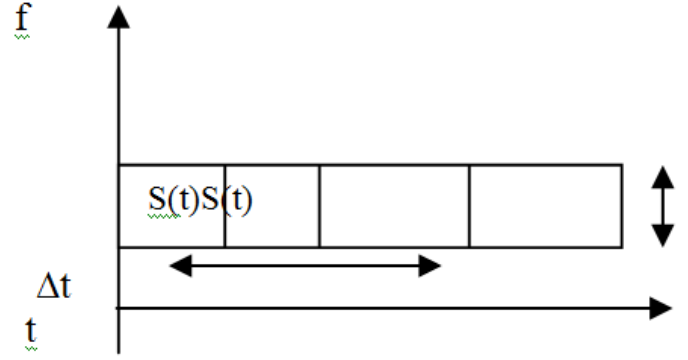

Fig.4 Time diversity

This technique is mostly used in fast fading environment where the coherence time of the channel is small.For slow fading environment, a large time delay is required, which may create significant problems for delay sensitive applications such as voice transmission [27].Awoseyila et.al[28] proposed an improved time diversity technique for short transmission time interval (TTI)and achieve significant time diversity gain in a wideband fading channel without reduction in the user data rate. Papaleo et.al [29].

\subsubsection{SPACE DIVERSITY}

Antenna diversity technique is also called space diversity. In space diversity two or more antenna can be used at the transmitter or receiver to improve the quality and reliability of a wireless system [30].This technique basically used in urban and indoor area.. When signal is effected due to fading this technique is used to remove that fading which is present in the signal due to multipath propagation. When more than two antenna are used at the receiver, this is called receive diversity and more than two antenna are used at the transmitter, is called transmit diversity. Which is shown in Fig. 5 and Fig.6[31],[23].

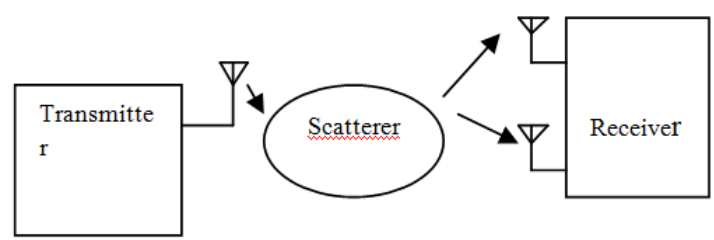

Fig.5 Receive diversity

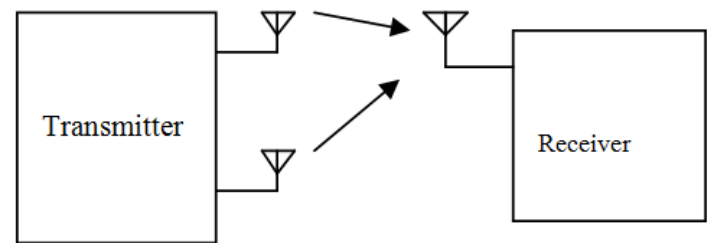

Fig.6 Transmit diversity

In receive diversity the independent fading paths associated with multiple receive antennas are combined to obtain a resultant signal which is passed through a standard demodulator. The major problem with using the receive diversity approach is the cost, size and power of the remote units. The use of multiple antennas and radio frequency (RF) chains (or selection and switching circuits) makes the remote units larger and more expensive. As a result diversity techniques have been applied to base stations to improve their reception quality. A base station often serves hundreds to thousands of remote units. 
Therefore it is more economical to add equipment to base stations rather than the remote units. For this reason transmit diversity schemes are very attractive and in transmit diversity there are multiple transmit antennas with the transmit power divided among these antennas. Transmit diversity is desirable in systems such as cellular systems where more space, power, and processing capability is available on the transmit side[32]. Wittneben et.al[33]proposed a delay diversity scheme for base station simul casting[34]. Seshadri et.al[35]suggested a similar scheme for a single base station in which copies of the same symbol were transmitted through multiple antennas at different times, hence it create an artificial multipath distortion[36]. Alamouti et. $\mathrm{Al}[32]$ proposed a new transmit diversity scheme using two transmit antennas and one receive antenna, the new scheme provides the same diversity order as maximal-ratio receiver combining (MRRC) with one transmit and two receive antennas. The disadvantage of space diversity is that it requires significant antenna separation, at least in the very high frequency and lower ultra high frequency bands. In the case where multipath signals arrive from all directions, antenna spacing should be in the order of $.5 \lambda$ to $.8 \lambda$ for independent or decor related channels.

\subsubsection{POLARIZATION DIVERSITY}

The space diversity technique is often used in the base station but polarization technique requires two horizontally separated antennas per sector. It contains a dual polarized antenna, in which only one antenna per sector is required shown in Fig[7]. The main polarization in the two branches of the antenna system is oriented in the $+/$ - 45 degrees direction or aligned in the horizontal/vertical-0/90 degrees directions [37].

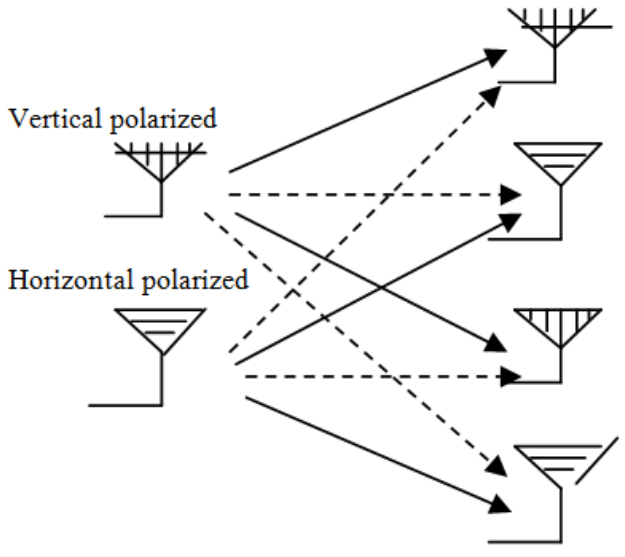

Fig.7 Polarization diversity

In this technology the electric and magnetic fields of the signal carrying the information are modified and many such signals are used to send the same information. Thus orthogonal type of polarization is obtained. Polarization diversity is used as an alternative as the same antenna can be used for different polarizations [38].Smith et.al [39] found that polarization diversity showed a marginally better performance than space diversity for the compact configurations used and it offers a very compact diversity arrangement with good antenna isolation. Buzzi et.al [40] focused on the use of polarization diversity techniques for multiuser non-orthogonal multipulse modulation and observed that polarization diversity was suitable to improve detection performance in wireless mobile communications. Correal et.al [41] proposed receiver architecture for the uplink of a DS-CDMA system and showed that the effects of flat fading could be significantly mitigated by combining multistage interference cancellation and polarization diversity. Fukushig et.al [42] proposed the analytical method for base station (BS) polarization diversity gain in a WCDMA system. The feature of this method wasthat the diversity gain would be treated as a transmission power value, which increases or decreases when Transmit Power Control (TPC) was applied [43].

\subsection{DIVERSITY COMBINING METHODS}

Diversity combining methods is used to combine several copies of the transmitted signal, which contain deep fading[44].The three types of diversity combining methods are discussed here [45].

\subsubsection{SELECTION COMBINING}

In selection combining, the branch that contains the largest signal-to-noise ratio is selected at any time at the receiver from a collection of antennas and the output of the receiver is connected to the demodulator, as shown in Fig.8 [46].

The signal to noise ratio at the input of each diversity branch is the ratio of instantaneous signal power to noise power.

$$
\begin{aligned}
& S N R_{P_{S}}=\max \left(S N R_{P_{1}}, S N R_{P_{2}}\right)=\max \left(\frac{r_{1}^{2}}{N}, \frac{r_{2}^{2}}{N}\right)=\frac{1}{N} \max \left(r_{1}^{2}, r_{2}^{2}\right) \\
& S N R_{V_{S}}=\sqrt{S N R_{P_{S}}}=\max \left(\frac{r_{1}}{\sqrt{N}}, \frac{r_{2}}{\sqrt{N}}\right)=\frac{1}{\sqrt{N}} \max \left(r_{1}, r_{2}\right)(5) \\
& \text { Selection combining output in (dB) = } \\
& \max \left(S N R_{1}, S N R_{2}\right)
\end{aligned}
$$

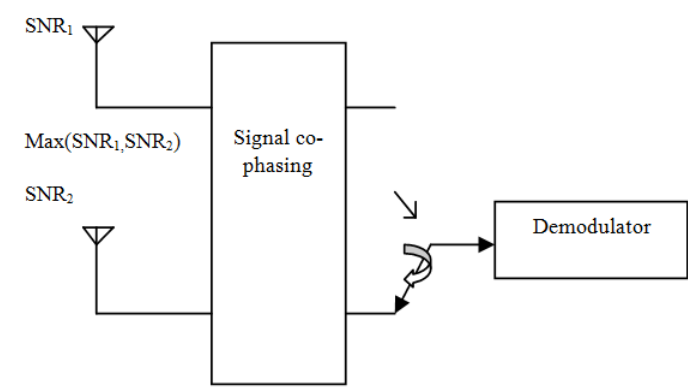

Fig.8 Block diagram of a two-branch selection combining system for equal noise powers in both branch

\subsubsection{MAXIMUM RATIO COMBINING}

In maximum ratio combining(MRC), each of the branch signals is weighted with a gain factor which is proportional to its own SNR. Then co-phasing and summing is done and summed signal is connected to the receiver and output signal of the receiver is connected to the demodulator. Shown in Fig.9[47]. In a Rayleigh fading channel MRC performance is best followed by equal gain combining and then selection diversity[48]. 
5.2.3 EQUAL GAIN COMBINING

In Equal gain combining (EGC) the outputs of different diversity branches are first co-phased and equally weighted and after that summing is done and summed signal is connected to the receiver and output signal of the receiver is connected to the demodulator [49].

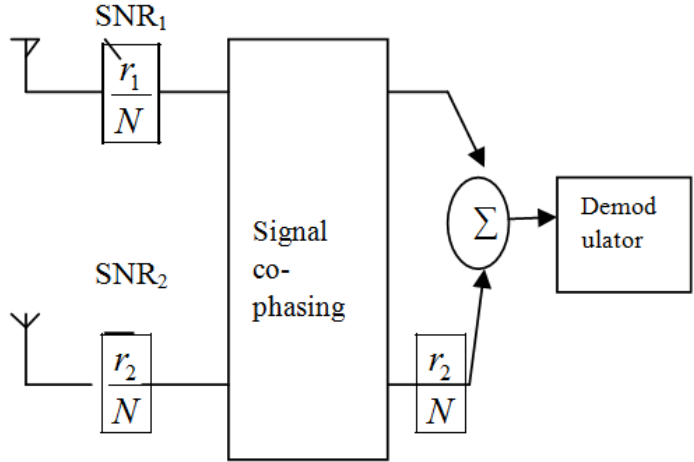

Fig.9 Block diagram of a two-branch maximal ratio combiner for equal noise powers in both branches

$$
\begin{gathered}
S_{N R_{P_{M}}\left(t_{0}\right)=} \frac{r_{1}\left(t_{0}\right)^{2}+r_{2}\left(t_{0}\right)^{2}}{N}=\frac{1}{N}\left(r_{1}\left(t_{0}\right)^{2}+r_{2}\left(t_{0}\right)^{2}\right) \mathrm{MRC} \\
\text { output }(\mathrm{dB})=S N R_{1}+S N R_{2} \\
\operatorname{SNR}_{V_{M}}\left(t_{0}\right)=\sqrt{S N R_{P_{M}}\left(t_{0}\right)}=\frac{1}{\sqrt{N}} \sqrt{r_{1}\left(t_{0}\right)^{2}+r_{2}\left(t_{0}\right)^{2}}
\end{gathered}
$$

EGC can be viewed as a special case of MRC which is shown in Fig.10.Ngamjaroen et.al[50] studied the comparison between co-phase and non co-phase EGC diversity techniques and the performance of non co-phase EGC diversity through the problem of limited space on wireless LAN terminal.

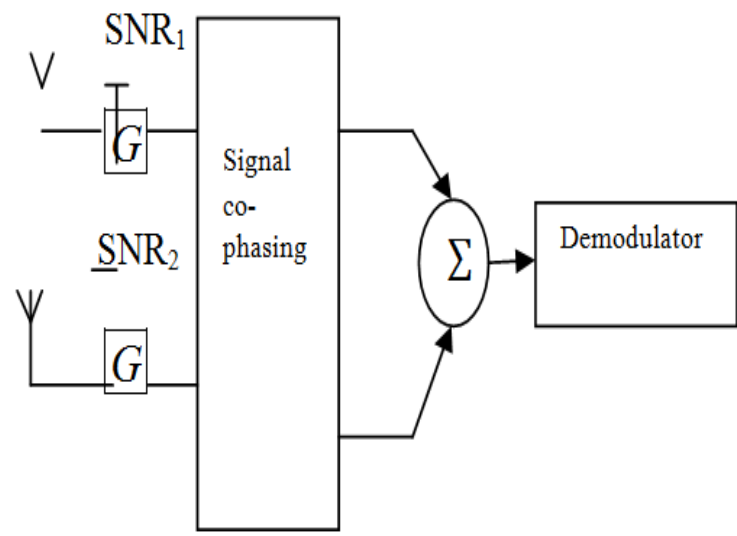

Fig.10Block diagram of a two-branch equal gain combiner for equal noise powers in both branches

$$
\begin{gathered}
S N R_{P_{E}}\left(t_{0}\right)=\frac{1}{2 N}\left(r_{1}\left(t_{0}\right)+r_{2}\left(t_{0}\right)\right)^{2} \\
\text { EGC output }(\mathrm{dB})= \\
0.5 S N R_{1}+0.5 S N R_{2}+\sqrt{S N R_{1}} \cdot \sqrt{S N R_{2}} \\
S N R_{V_{E}}\left(t_{0}\right)=\sqrt{S N R_{P_{E}}}=\frac{1}{\sqrt{2 N}}\left(r_{1}\left(t_{0}\right)+r_{2}\left(t_{0}\right)\right)
\end{gathered}
$$

\section{CONCLUSION}

For high data rate transmission WCDMA is used and but performance of WCDMA is limited by fading and other factors such as interference, scattering etc. Different diversity techniques are used to mitigate fading. In this paper different diversity techniques are reviewed to reduce the fading problem. In all diversity techniques maximum ratio combining is mostly used due to high diversity gain and high signal to noise ratio compare to other diversity techniques.

\section{REFERENCES}

[1]. Holma H. and Toskala A. "WCDMA for UMTS, "John Wiley \& Sons Ltd,(2002).

[2]. Wang X., "Wireless communication systems: advanced techniques for signal reception", Prentice,(2004)

[3]. http://wireless.per.nl/reference/chaptr01/telephon/is54.htm

[4]. ETSI EN 300 910, "Digital cellular telecommunications system (Phase2+); radio transmission and reception," GSM 05, Version 8.5.1 Release 1999,(2000).

[5]. Tan D.K.P., Sun H.and Lu.Y, "Passive radar using global system for mobile communication signal: theory, implementation and measurements", IEEE Journal on Rader, Sonar and Navigation, Vol. 152,No.3, pp 116-123, (2005).

[6]. Rappaport T.S., Wireless Communication, Principles \& Practice, Prentice Hall, Upper Saddle River, NJ, $2^{\text {nd }}$ edition, C.(2002).

[7]. Peterson R.L., Ziemer R.E. and Borth D.E., "Introduction to spread spectrum communications", Prentice-Hall, (1995).

[8]. Jung P., Baier P. W. and Steil A., "Advance of CDMA and spread spectrum techniques over FDMA and TDMA in cellular mobile radio applications", IEEE Transactions on Vehicular Technology, Vol. 42, No. 3, pp. 357-64, (1993).

[9]. Milstein L.B, "Wideband code division multiple access", IEEE Journal on selected areas in communication, Vol-18, No.8,pp-13441354, (2000)

[10]. Olavarrieta L.D., "Wireless communications education: A Guide to Important Topics,Microwave Review, (2005)

[11]. Prasad R. and Ojanpera T., "An overview of CDMA evolution towards wideband CDMA," IEEE communication survey, Vol.1,pp.2-29, fourth quarter (1998).

[12]. Dahlman E, Gudmundson B, ilsson M. and Skold J. “ UMTS/IMT-2000 Based on Wideband CDMA,"IEEE Communications Magazine, Vol. 36, pp. 70-80, (1998).

[13]. Dahlman E., Beming P., Knutsson J., Ovesjo F.,Persson M. and Roobol C. "WCDMA- The Radio Interface for Future Mobile Multimedia Communications," IEEE Transactions on Vehicular Technology, Vol. 47, No. 4, pp. 1105-1118, (1998).

[14]. ETSI TS125301 V3.3.0. Universal mobile telecommunications system (UMTS); Radio Interface Protocol Architecture,(1999).

[15]. Ojanpera T. and Prasad R. "Wideband CDMA for Third Generation Mobile Communications," Artech House, Boston, London,(1998)

[16]. William C.Y.,"Mobile communications engineering theory and applications", Second edition, Tata Mc Graw Hill Publishing company limited, New Delhi, (2008).

[17]. Stallings W, "Wireless Communications and Networks", 2nd ed., Upper Saddle River,NJ: Prentice-Hall, (2005).

[18]. Mayer T. , Robertson, C. , Ha, T.T. :" Co-channel interference reduction on the forward channel of a wideband CDMA cellular system" in Proceedings of IEEE International Conference on Military Communications, Vol.2, pp, 785 - 790 ,(1999).

[19]. Abderrahmane N.; Merouane M.:"Diversity Techniques to combat fading in WiMAX," ISSN: 1109-2742, Vol.7,No.2 (2008).

[20]. Kadhim M.A. and Ismail W.,"Performation diversity techniques for wireless communication system", journal of telecommunications, vol. 4, issue 2, (2010).

[21]. Srivastava N., "Diversity schemes for wireless communication a Short review," journal of theoretical and applied information technology,islamabad pakistan, Vol.15. No.2,(2010).

[22]. Ziemer R.E. and Petterson R.L.: Introduction to Digital Communication, $2^{\text {nd }}$ edition, Prentice Hall, (2000)

[23]. Vucetic B. and Yuan J.: Space-Time CodingWiley, (2003). 
[24]. Xiaoya Z; Dong X and Yongsheng W."Time domain spreading and frequency domain maximal ratio combining reception for frequency diversity enhancement in single carrier UWB communication systems" Can. J. Elect. Comput. Eng., Vol. 34, No. 4, (2009).

[25]. Li J. and Liu X. “ A Frequency diversity technique for interference mitigation in coexisting bluetooth and WLAN" pp 5490 - 5495 , 14244-0353-7, (2007)

[26]. Proakis J.G.: Digital Communications, McGraw-Hill, (1995).

[27]. Gordon S.L., "Principle of mobile communication," Kluwer academic, boston, (1996)

[28]. Awoseyila A. and Evans B. "Improved time diversity for LTE over satellite using split multicode transmission" electronics letters Vol. 46 No. 10 , (2010).

29]. Papaleo M., Neri M., Vanelli-Coralli A. and Corazza. G.E. "Using LTE in 4G satellite communications: increasing time diversity through forced retransmission". 10th Int. Workshop on Signal Processing Space Communications, Rhodes Island, Greece, pp. 1-4, (2008).

[30]. Tepedelenlioglu C. and Gao P., "On Diversity Reception over fading channels with impulsive noise," IEEE Transactions on Vehicular Technology, Vol. 54, Issue 6, pp 2037-2047, (2005).

[31]. Jakes W.C., Microwave Mobile Communications.New York: Wiley, (1974).

[32]. Alamouti S.M., "A Simple Transmit Diversity Technique for Wireless communications," IEEE journal on select areas in communications, Vol. 16,No. 8, pp 1451-1458,(1998).

[33]. Wittneben A., "Base station modulation diversity for digital simulcast," in Proc. 1991 IEEE Vehicular Technology Conf. pp. 848-853,(1993).

[34]. Wittneben A., "A new bandwidth efficient transmit antenna modulation diversity scheme for linear digital modulation," in Proc. 1993 IEEE International Conf. Communications,pp. 1630-1634, (1993)

[35]. Seshadri N. and Winters J.H, "Two signaling schemes for improving the error performance of FDD transmission systems using transmitter antenna diversity," in Proc. 1993 IEEE Vehicular Technology Conf., pp. 508-511, (1993).

[36]. Winters J.H., "The diversity gain of transmit diversity in wireless systems with Rayleigh fading," in Proc. 1994 icc/supercomm, New Orleans, LA, Vol. 2, pp. 1121-1125, (1994).

[37]. Brown T., "Characterization of polarization diversity at the mobile", IEEE Transactions on Vehicular Technology, vol. 56, No. 5, (2007).

[38]. Yang H.and Alouini M. "MRC \& GSC Diversity Combining with an output threshold," IEEE Transactions on Vehicular Technology, vol. 54, issue R. Yates, A frame work for uplink Power control in cellular radio systems", IEEE Journal on selected areas in comm., Vol. 13, issue 7, pp. 1341-1347, (2005).

[39]. Smith M. and Neal L." A Comparison of Polarisation and Space Diversity for Indoor Propagation at $900 \mathrm{MHz}$,IEEE 0-7803- 1396, 1993.

[40]. Buzzi S.,Conte E. and Maio A.,"Polarisation diversity reception of nonorthogonal multipulse signals in multiuser rayleigh fading channels"IEEE proc-communication Vol. 150, No 2, (2003)

[41]. Correal N.S. and Woerner B.D. "Enhanced DS-CDMA uplink performance through base station polarization diversity and Multistage interference cancellation" Proceedings of 1998 IEEE Global telecommunications conference , Vol. 4.pp 96-100, DIJ.1905-1910,(1998).

[42]. Fukushige M.and Imai T.,"Analysis of polarization diversity gain at base station in WCDMA system",International Symposium on antennas and propagation, ISAP (2006).

[43]. Kozono S.; Tsuruhara T. and Sakamoto M., "Base station polarization diversity reception for mobile radio," IEEE Trans. VT, Vol.-33, No. 4, pp. 301-306, (1984).

[44]. Nuaymi L.; Godlewski P and Lagrange X., "Power Allocation Control for the Downlink in Cellular CDMA Networks". In Proceeding of the 12th IEEE International Symposium on Personal, Indoor and Mobile Radio Communications (PIMRC'01), vol.1,pagesC29-C31, San Diego, CA, (2005).

[45]. Bonds H. , "System performance in fading channel environments", M.S.E.E, (2003)

[46]. Neasmith E.A and Beaulieu N.C., "New results on selection diversity", IEEE Transactions on Communications, Vol.46,pp 695704,(1998).
[47]. Zhou h. and Okamoto k., "Comparison of code combining and MRC diversity reception in mobile communications", IEEE Wireless Communications and Networking Conference, Atlanta, USA,Vol.2,pp 908-913, (2004).

[48]. Bolcskei H. and Paulraj A., " Multiple-input multiple -output (MIMO) wireless systems," Chapter in " The Communication Handbook", 2nd edition, J. Gibson, Ed., CRC Press, pp. 90.190.14, (2002)

[49]. Alouini M.S. and Simon M.K., "Performance Analysis of Coherent Equal Gain Combining over Nakagamim Fading Channels," IEEE Transactions on Vehicular Technology, Vol. 50,pp. 1449-1463, (2001).

[50]. Ngamjaroen T. and Uthansakul.P,"Performance of Non Co-phase EGC Diversity Technique with Multiple Antennas on Limited Space" ecti transactions on electrical eng., electronics, and communications ,Vol.9, No.1, (2011) 